\title{
Ion Mobility Spectrometer with Orthogonal X-Ray Source for Increased Sensitivity
}

Authors: Erik Bunert, Tobias Reinecke, Ansgar T. Kirk, Alexander Bohnhorst, Stefan Zimmermann

Affiliation: Leibniz Universität Hannover, Institute of Electrical Engineering and Measurement Technology, Department of Sensors and Measurement Technology, Appelstr. 9A, 30167 Hannover, Germany

Corresponding Author: Erik Bunert, bunert@geml.uni-hannover.de

\begin{abstract}
Ion mobility spectrometers (IMS) are compact devices for extremely sensitive detection of proton and electron affine volatile compounds down to low $\mathrm{ppt}_{\mathrm{v}}$ concentrations within less than a second. The measuring principle requires ionization of the target analyte. Most IMS employ radioactive electron sources, such as ${ }^{63} \mathrm{Ni}$ or ${ }^{3} \mathrm{H}$. These radioactive materials suffer from legal restrictions limiting the fields of application. Furthermore, the electron emission has a predetermined intensity and cannot be controlled or disabled. In a previous work, we replaced the axially mounted ${ }^{3} \mathrm{H}$ source of our ion mobility spectrometer with a commercially available X-ray source operated at low acceleration voltage of $4.5 \mathrm{kV}$ to be applicable in most application without legal restrictions. However, the high penetration depth of the radiation together with the statistical behavior of the X-ray ionization process led to an increase of Fano noise and thus a limited signal-to-noise ratio. Therefore, the X-ray source is now mounted orthogonal to the drift tube in order to avoid Fano noise. Here, we compare the analytical performance of this orthogonal setup with the axially mounted X-ray source. The noise level is significantly reduced. This improves the signal-to-noise ratio from 700 with the axially placed source to more than 3000 with the orthogonally placed source, while the resolving power still remains at $R=100$. Furthermore, typical limits of detection for some model substances in the low $\mathrm{ppt}_{\mathrm{v}}$ range in positive and negative ion mode are given.
\end{abstract}

Keywords: Ion Mobility Spectrometry; X-ray IMS; orthogonal X-ray source; X-ray ionization; nonradioactive ionization source.

\section{Introduction}

Drift tube ion mobility spectrometers (IMS) are well-known devices for extremely sensitive detection of proton and electron affine volatile compounds down to low ppt $_{v}$ concentrations within less than a second. By ionizing the analyte in the ionization region and subsequently gating the ions into the drift region by an ion shutter, the different ion species can be separated by their mobility in a neutral gas under the influence of an electric field [1]. With its acceptable separation power and a comparatively compact design, IMS are commonly used in safety and security applications such as the detection of toxic industrial compounds [2], drugs [3], explosives [3,4] and chemical warfare agents [4,5]. However, the required initial ionization of the analyte is commonly initiated by high kinetic energy electrons, emitted from a radioactive beta minus decay, such as from ${ }^{3} \mathrm{H}$ or ${ }^{63} \mathrm{Ni}$. The disadvantages of these radioactive electron sources are legal restrictions affecting import, export, deployment in certain areas and disposal of such devices. Furthermore, the electron emission can neither be controlled nor disabled. Therefore, the interest in non-radioactive alternatives has grown in recent years. Possible alternatives are e.g. UV photoionization lamps [6,7], corona discharge [8-10] or non-radioactive electron sources [11,12]. Unfortunately, the generated spectra by UV photoionization (Atmospheric pressure photo ionization, APPI) and corona discharge differs from the spectra of a radioactive electron source (Atmospheric pressure chemical ionization, APCI), so direct 
comparison of data is difficult [6,9]. In addition, APPI is a direct ionization and therefore often less sensitive than $\mathrm{APCl}$, especially for proton and electron affine compounds. Non-radioactive electron sources generate comparable spectra, but are not commercially available as original equipment manufacturer (OEM). Therefore, in a previous work [13] we investigated the performance of an X-ray source as a replacement of the radioactive tritium source in our IMS [14]. We found that the X-ray source generates comparable spectra with a comparable resolving power $[13,15]$, which leads to the same applications as those of conventional IMS with radioactive source. However, the disadvantage of the X-ray source is a very high penetration depth, which results in a constant ionization of the drift gas inside the drift region. This results in an offset current in the spectrum depending on the source activity. While the signal intensity increases with higher source activity, the increased offset current leads to increasing noise in the spectrum. The increasing noise has been attributed to Fano noise [16], which is induced by the statistical behavior of the Xray ionization process and depends on the number of produced ions. Thus, the maximum achievable signalto-noise ratio (SNR) is limited to the point where the increase in signal amplitude is lower than the increase in noise. In order to achieve a significant improvement of sensitivity, setup modifications are required to avoid ionization of the drift gas in the drift region [13]. In this work, the IMS design is modified by arranging the X-ray source orthogonal to the drift tube, to focus the X-ray beam to the ionization region. Furthermore, we investigate the influence of this modification on the analytical power of the IMS and compare it with the axial arrangement.

\section{Experimental}

To exclusively investigate the influence of the X-ray source position on the analytical power of the IMS, a universal source mounting for two positions is required. Therefore, we designed a new source mounting modifying the design of our previously described current drift tube [17] with an increased drift length of $83.5 \mathrm{~mm}$ to accommodate the orthogonal mounting. The current drift tube is already an improved version of the drift tube used in our previous X-ray IMS $[13,14]$, thus providing higher SNR. Figure 1 depicts a schematic representation of the two possible source positions. The source can be arranged in either axial or orthogonal orientation, as shown in Fig. 1 (a) and (b). These two source mountings are both coupled to the modified IMS, as shown in Fig. 1 (c), so that the X-ray source can be easily changed from the axial to the orthogonal position.

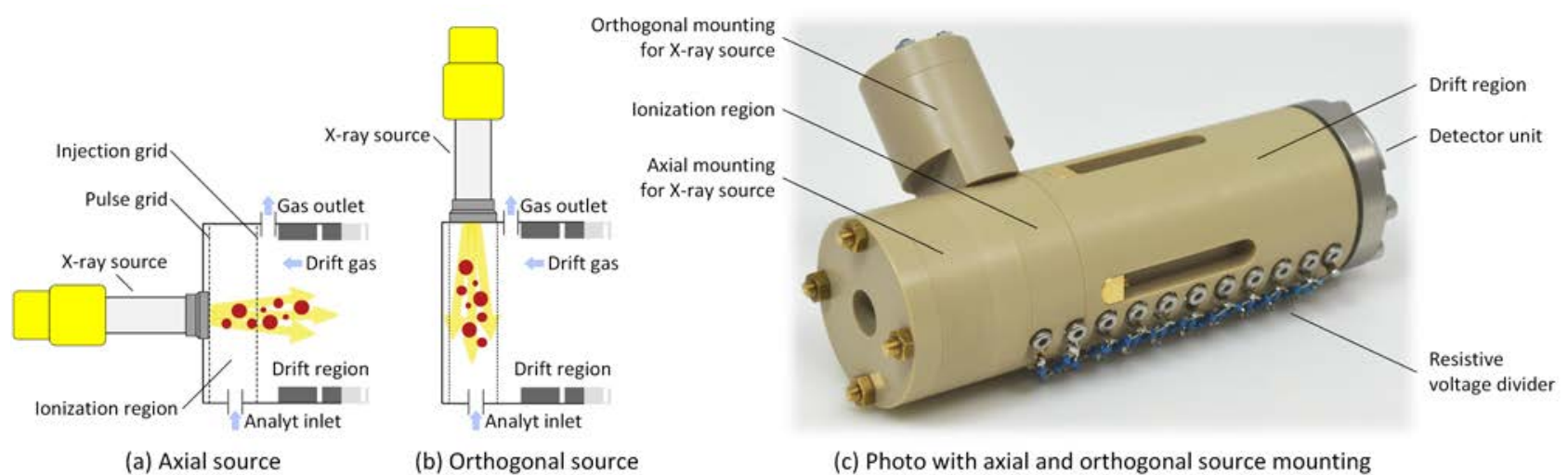

Fig. 1. Schematic representation of the ionization region with axially (a) and orthogonally (b) mounted X-ray source. Both source mountings are simultaneously coupled to our modified IMS (c).

The remaining setup used in this work consists of a commercially available miniature X-ray source with rhodium target (XRT-50-2-Rh-0.6-125, Newton Scientific, Inc., USA), which was already used in our previous work [13], an ionization region, a drift region and a Faraday plate detector with aperture grid. The ionization and drift regions are separated by an injection grid. The ion shutter pushes the generated ions into the drift 
region. For this purpose, a Bradbury-Nielsen shutter is typically used in many applications $[1,18]$. Alternatively, we employ a field switching shutter, which is easier to manufacture, and has better stability and reduced distortions of the drift field [18]. The latter is the key for injecting short ion packets to reach high resolving power. During the injection time of typically $t_{\text {inj }}=20 \mu \mathrm{s}$, the potential at the pulse grid is $500 \mathrm{~V}$ above the injection grid (positive ion injection). Thus, the ions are pushed into the drift region as a small ion packet.

To achieve a homogeneous electrical field in the drift region, the drift voltage of $U_{\text {drift }}=4.93 \mathrm{kV}$ is applied via a resistive voltage divider to stainless steel ring electrodes separated by polyether ether ketone (PEEK) isolation rings. The injected ions drift towards the detector under the influence of this electrical field. Thereby, the different ion species are separated by their specific mobility. At the end of the drift regions the separated ions discharge on the Faraday detector and the resulting ion current is plotted over the drift time to obtain the ion mobility spectrum. The drift region is purged with purified dry air with a constant flow of $150 \mathrm{ml}_{\mathrm{s}} / \mathrm{min}$ (milliliter standard per minute, mass flow at reference conditions $20^{\circ} \mathrm{C}$ and $1013.25 \mathrm{hPa}$ ) and a dew point of $-89^{\circ} \mathrm{C}$. All operating parameters are listed in Table 1.

Table 1: IMS and X-ray source operating parameters.

\begin{tabular}{|c|c|}
\hline Parameter & Value \\
\hline Drift length $/_{\text {drift }}$ & $83.5 \mathrm{~mm}$ \\
\hline Drift region diameter & $21 \mathrm{~mm}$ \\
\hline Repetition rate & $40 \mathrm{~Hz}$ \\
\hline Drift voltage $U_{\text {drift }}$ & $4.93 \mathrm{kV}$ \\
\hline Drift gas flow & $150 \mathrm{ml}_{\mathrm{s}} / \mathrm{min}$ \\
\hline Sample gas flow & $10 \mathrm{ml}_{\mathrm{s}} / \mathrm{min}$ \\
\hline Dew point drift gas & $-89^{\circ} \mathrm{C}$ \\
\hline Operating pressure & $1018 \mathrm{hPa}$ \\
\hline Operating temperature & $30^{\circ} \mathrm{C}$ \\
\hline $\begin{array}{l}\text { X-ray source } \\
\text { acceleration voltage } \\
U_{\text {acc }}\end{array}$ & $4.5 \mathrm{kV}$ \\
\hline $\begin{array}{l}X \text {-ray source filament } \\
\text { current } I_{\text {fil }}\end{array}$ & $500 \mathrm{~mA} \ldots 620 \mathrm{~mA}$ \\
\hline $\begin{array}{l}\text { X-ray source emission } \\
\text { current } I_{\text {emis }}\end{array}$ & $1 \mu \mathrm{A} \ldots 25 \mu \mathrm{A}$ \\
\hline
\end{tabular}

We have characterized different model substances, purchased from Sigma Aldrich. For this purpose, the different substances are filled in a vial heated to $35^{\circ} \mathrm{C}$ in a permeation oven, except for 1-octanol, which was heated to $40^{\circ} \mathrm{C}$ for better permeation. For generating a constant analyte vapor concentration, the oven is purged with a constant flow of $600 \mathrm{ml}_{\mathrm{s}} / \mathrm{min}$ of purified dry air. An adjustable fraction of this gas is diluted with dry air for generating different analyte concentrations in the sample gas. 


\section{Comparison of the analytical performance between axial and orthogonal X-ray source position}

This section describes the improvements in analytical performance of the IMS with orthogonally mounted $\mathrm{X}$-ray source and axial source position. As previously mentioned, the major disadvantage of the axially mounted $X$-ray source is the increased Fano noise, arising from the high penetration depth of the radiation and hence ionization of the neutral gas inside the drift region. To investigate the influence of source orientation on the Fano noise, the SNR measured as the ratio of the offset-corrected peak height of the positive reactant ion peak $\left(\mathrm{RIP}^{+}\right)$to the noise $\sigma$ is determined for different filament currents and for both source positions. The corresponding ion mobility spectra for the axial position are shown in Fig. 2 (a) for four selected filament currents leading to $\mathrm{RIP}^{+}$amplitudes of about $250 \mathrm{pA}, 700$ pA, 1000 pA and 1500 pA.
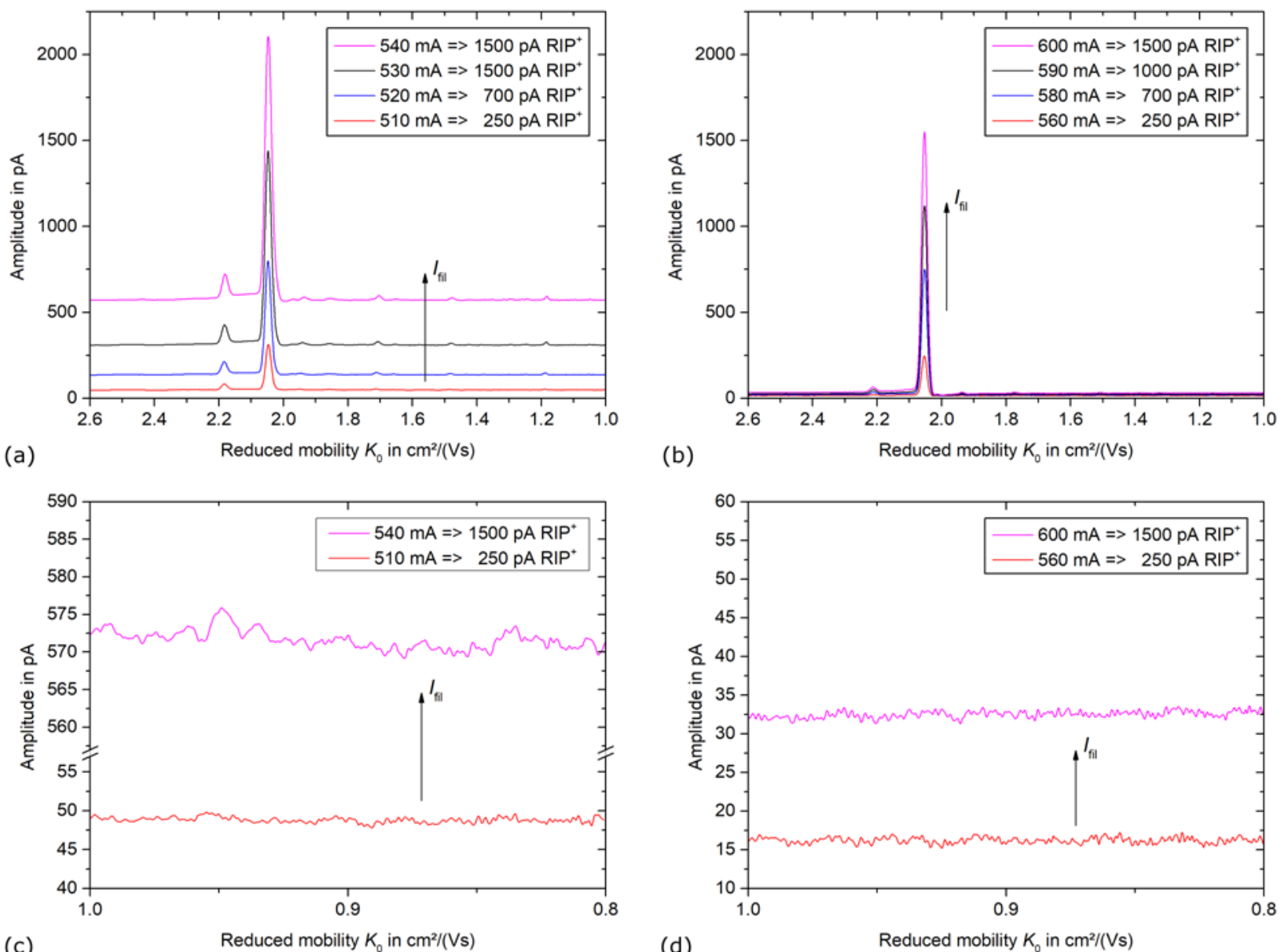

Fig. 2. Ion mobility spectra of purified dry air (dew point of $-89^{\circ} \mathrm{C}$ ) for different filament currents $I_{\text {fil }}$ for axial (a) and orthogonal (b) orientation of the X-ray source leading to RIP ${ }^{+}$amplitudes of about $250 \mathrm{pA}, 700 \mathrm{pA}, 1000 \mathrm{pA}$ and $1500 \mathrm{pA}$. The dominant peak is the positive reactant ion peak $\left(\mathrm{RIP}^{+}\right)$at $K_{0}=2.05 \mathrm{~cm}^{2} \mathrm{~V}^{-1} \mathrm{~s}^{-1}$. The noise level of the axial setup (c) significantly increases with higher RIP ${ }^{+}$amplitudes, the noise level of the orthogonal setup (d) remains nearly constant.

Typically, the drift time is replaced by the ion mobility $K$. The ion mobility can be normalized for standard conditions $\left(T_{0}=273.15 \mathrm{~K}\right.$ and $p_{0}=1013.25 \mathrm{hPa}$ ) leading to the reduced ion mobility $K_{0}[4,19]$. The RIP ${ }^{+}$is located at a reduced ion mobility of $K_{0}=2.05 \mathrm{~cm}^{2} \mathrm{~V}^{-1} \mathrm{~s}^{-1}$. As expected, the increasing filament current leads to an increased $\mathrm{RIP}^{+}$amplitude and an increased offset current of the spectrum. However, when using the orthogonal source position, an increased filament current also leads to an increased RIP $^{+}$amplitude but with almost no increase in offset current, as shown in Fig. 2 (b). Therefore, the ionization process in the drift 
region can, as expected, be significantly reduced by mounting the X-ray source orthogonally to the drift tube. Thus, the noise of the baseline of the spectrum can also be significantly reduced for high RIP amplitudes with the orthognal setup, as shown in Fig. 2 (c) and (d). However, higher filament currents are required to obtain the same $\mathrm{RIP}^{+}$amplitudes as in the axial position. This is due to the small dimensions of the ionization region and thus the resulting smaller radiation cross-section in the orthogonal position.

The focus of the following investigations is on the limit of detection (LoD) for different analytes. Here, the LoD is defined as three times the standard deviation $(3 \sigma)$ of the noise level. Thus, when decreasing the noise level, the LOD can be improved. To compare the noise of the two considered configurations, Fig. 3 shows the variance $\left(\sigma^{2}\right)$ of the noise after averaging for $1 \mathrm{~s}$ as a function of the offset current. The standard deviation of the noise $(\sigma)$ is determined for drift times between $15 \mathrm{~ms}$ and $17 \mathrm{~ms}$ of the respective spectrum (respectively low reduced mobilities $K_{0}$ between $0.86 \mathrm{~cm}^{2} \mathrm{~V}^{-1} \mathrm{~s}^{-1}$ and $0.75 \mathrm{~cm}^{2} \mathrm{~V}^{-1} \mathrm{~s}^{-1}$ ), where typically no analyte peaks are present. The linearly increasing variance with increasing offset current shows that the noise is dominated by the Fano noise [16]. As shown by the inset in Fig. 3, both the axially and the orthogonally mounted source exhibit the same relationship between offset current and noise. However, the generated offset current at the same filament current is significantly smaller when using the orthogonal source position. Therefore, with the orthogonally mounted source only tiny offset currents can be investigated.

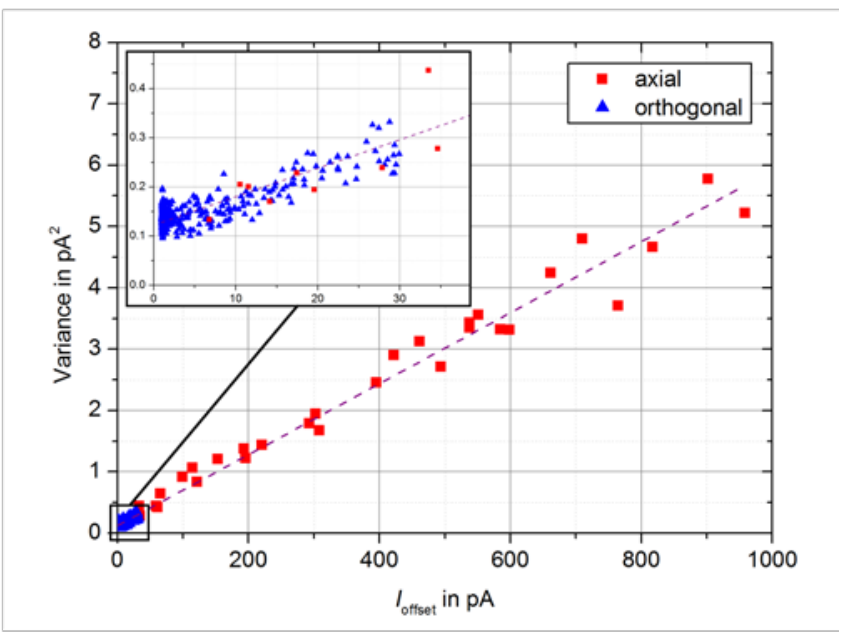

Fig. 3. Variance of noise in the spectrum vs. offset current loffset for axial (red squares) and orthogonal (blue triangles) orientation of the X-ray source.

Using the orthogonal setup, fewer ionization processes occur in the drift region, leading to the lower offset current and decreased Fano noise level at equal RIP ${ }^{+}$amplitudes, thus improving the SNR. For a more detailed investigation, Fig. 4 shows the signal-to-noise ratio (SNR) over the RIP ${ }^{+}$-amplitude. The SNR of the axially mounted X-ray source is limited to a value of less than SNR $=1000$, because at RIP ${ }^{+}$amplitudes of more than $1000 \mathrm{pA}$ the increase in amplitude is lower than the increase in noise. In contrast, the SNR with orthogonally mounted $\mathrm{X}$-ray source shows only a small saturation effect even for high intensities of the Xray source. Assuming a constant ionization efficiency, this increase in signal-to-noise ratio would translate to an equal decrease in limits of detection. The above conclusions do not consider other peaks in the ion mobility spectrum, e.g. caused by impurities, due to their relatively small amplitude. 


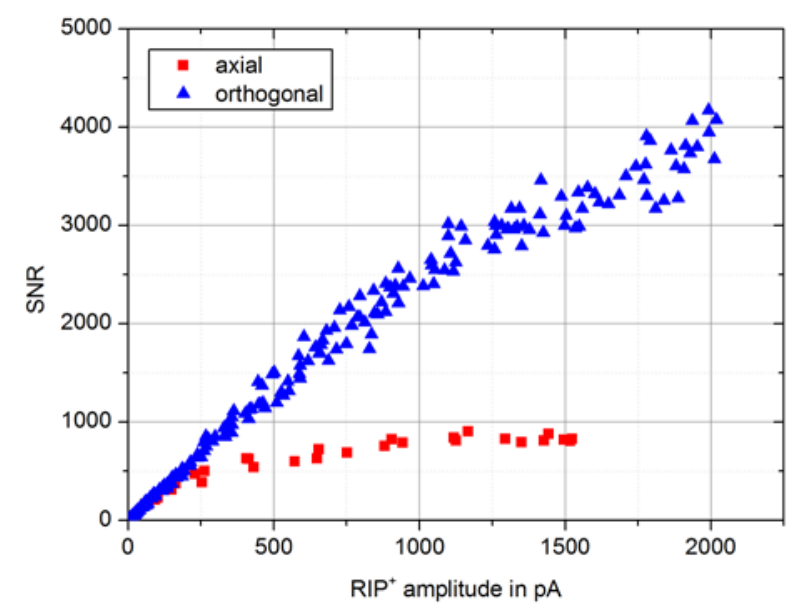

Fig. 4. Signal-to-noise ratio of the $\operatorname{RIP}^{+}$(SNR) vs. RIP ${ }^{+}$amplitude for axial (red squares) and orthogonal (blue triangles) orientation of the X-ray source.

Another key factor for a high analytical performance is an efficient separation of the different ion species [20]. In IMS, the resolving power $R$ is a good measure for separation power as $R$ is nearly independent of the peak position. It is defined as the ratio of the drift time $t_{\text {drift }}$ of the ion peak to its full width at half maximum (FWHM) $w_{0.5}$, see eqn. (1) [1].

$$
R=\frac{t_{\text {drift }}}{w_{0.5}}
$$

Thus, the higher the resolving power, the better ion species with similar ion mobilities can be separated from each other. The disadvantage of high peak amplitudes is a decreased resolving power because of the higher ion density and the resulting peak broadening by Coulomb repulsion. Therefore, we measured the resolving power as a function of the SNR. The measurement results in Fig. 5 show that the decrease in the resolving power with increasing SNR is significantly stronger when using the axially mounted X-ray source, compared to the orthogonal mounted source. For example, the typical resolving power of our IMS with a radioactive tritium source is $R=100$. Using the axial source, the resolving power already drops below this value at an SNR of 700. However, if the orthogonal source is used, the resolving power remains above this value until a SNR of 3000, since at the same SNR the RIP amplitude and thus the Coulomb repulsion is significantly smaller than with the axial setup.

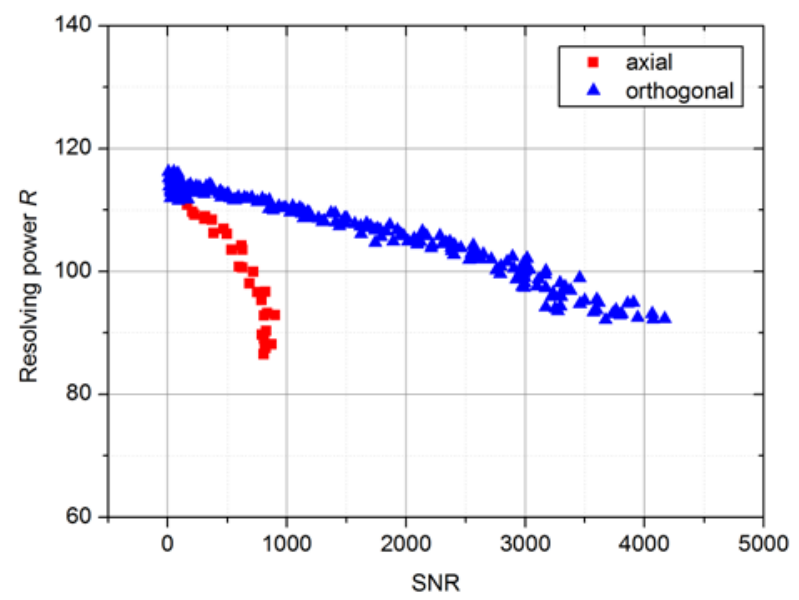

Fig. 5. Resolving power R vs. signal-to-noise ratio (SNR) for axial (red squares) and orthogonal (blue triangles) orientation of the $\mathrm{X}$-ray source. 
Therefore, with the orthogonal source, significantly lower detection limits can be achieved with only slightly reduced resolving power, which is not possible with the axial source. In addition, a choice can be made between a better limit of detection and a higher resolving power. The minimum attainable limits of detection with the orthogonally mounted X-ray source for some selected model substances are presented in the following. By an APCI process, the reactant ions react with the analyte molecules and the analyte ions are generated. By detecting the specific analyte ion peaks in the spectrum and measuring the ambient parameters, the corresponding reduced ion mobility can be determined. The polarity of the IMS can be switched to detect both ion polarities. For example, Fig. 6 (a) shows the positive spectrum for $10 \mathrm{ppb}_{\mathrm{v}}$ dimethyl methylphosphonate (DMMP) with the positive reactant ion peak $\left(\mathrm{RIP}^{+}\right)$, a DMMP monomer and a

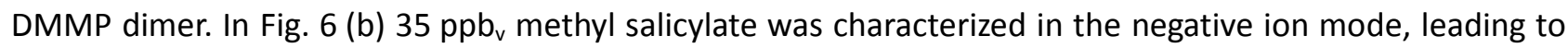
the negative reactant ion peak (RIP-) and a single methyl salicylate peak (monomer).
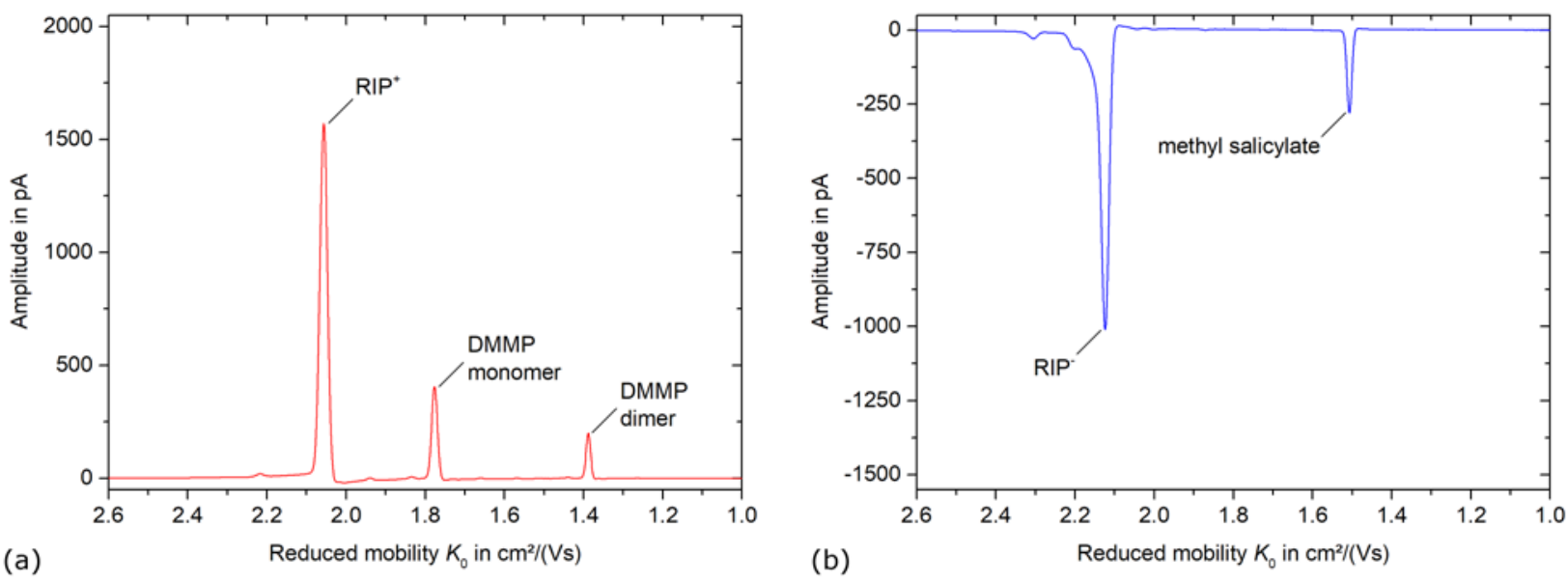

Fig. 6: Positive ion mobility spectrum of $10 \mathrm{ppb}_{v}$ dimethyl methylphosphonate (DMMP) (a) and negative ion mobility spectrum of $35 \mathrm{ppb}_{v}$ methyl salicylate (b) in clean dry air.

For all LoD measurements, we set the acceleration voltage of the $X$-ray source to $U_{\text {acc }}=4.5 \mathrm{kV}$ and the filament current to $I_{\text {fil }}=612 \mathrm{~mA}$ leading to an SNR for the RIP ${ }^{+}$of 4000 . Now, we calculated the LoD based on three times of the standard deviation $(3 \sigma)$ of the measured noise of the baseline and the measured linear amplitude increase of the respective analyte peak at low concentrations, as shown in Fig. 7 for the acetone monomer.

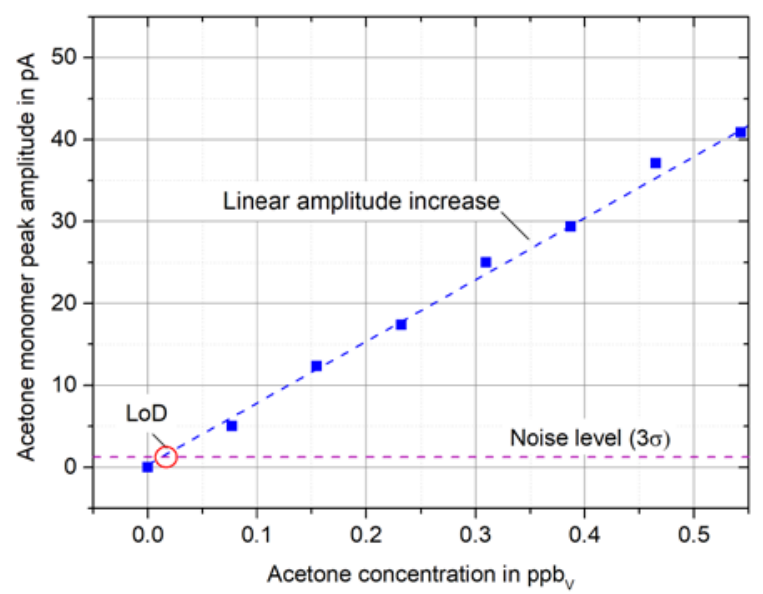

Fig. 7. Determination of the LoD for acetone monomer based on the intersection of the noise level ( $3 \sigma$ ) and the measured linear amplitude increase of the respective analyte peak. 
The calculated LoDs using the orthogonal setup for an averaging time of $1 \mathrm{~s}$ are listed in Table 2 . The selected model substances are from several substance classes and already investigated with our other IMS setups (e.g. IMS with radioactive ionization source). Compared to the setup with axially mounted X-ray source the limits of detection can be significantly improved by a factor of 4 , as expected from the improvement of the SNR. The limits of detection are also improved compared to our IMS when equipped with a radioactive tritium source, which are e.g. 60 ppt $_{v}$ for acetone monomer (positive ion mode) and $130 \mathrm{ppt}_{\mathrm{v}}$ for methyl salicylate (positive ion mode) [13].

Table 2: Calculated limits of detection (LoD) and reduced ion mobility values $\mathrm{K}_{0}$ for various substances with orthogonal arranged X-ray source.

\begin{tabular}{|c|c|c|c|c|}
\hline Substance & Polarity & 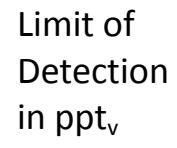 & $\begin{array}{l}\text { Reduced ion mobility } K_{0} \text { at } \\
1013.25 \mathrm{hPa} \text { and } 273.15 \mathrm{~K} \\
\text { in } \mathrm{cm}^{2} \mathrm{~V}^{-1} \mathrm{~s}^{-1}\end{array}$ & CAS Number \\
\hline $\mathrm{RIP}^{+}$ & Positive & - & 2.05 & - \\
\hline $\mathrm{RIP}^{-}$ & Negative & - & 2.14 & - \\
\hline 1-octanol (monomer) & & 230 & 1.37 & \multirow{3}{*}{$111-87-5$} \\
\hline 1-octanol (dimer) & \multirow[t]{2}{*}{ Positive } & 2,000 & 1.06 & \\
\hline 1-octanol (trimer) & & 5,700 & 0.87 & \\
\hline 1,1,2-trichloroethane & Negative & 80 & 2.39 & $79-00-5$ \\
\hline Acetone (monomer) & \multirow{2}{*}{ Positive } & 20 & 2.04 & \multirow{2}{*}{$67-64-1$} \\
\hline Acetone (dimer) & & 600 & 1.78 & \\
\hline Ammonia (monomer) & \multirow{2}{*}{ Positive } & 30 & 2.39 & \multirow{2}{*}{$506-87-6$} \\
\hline Ammonia (dimer) & & 7,500 & 2.22 & \\
\hline $\begin{array}{l}\text { Dimethyl } \\
\text { methylphosphonate } \\
\text { (DMMP) (monomer) }\end{array}$ & & 20 & 1.78 & \multirow[b]{2}{*}{$756-79-6$} \\
\hline $\begin{array}{l}\text { Dimethyl } \\
\text { methylphosphonate } \\
\text { (DMMP) (dimer) }\end{array}$ & Positive & 940 & 1.39 & \\
\hline $\begin{array}{l}\text { Isopropyl alcohol } \\
\text { (monomer) }\end{array}$ & \multirow[t]{2}{*}{ Positive } & 30 & 1.87 & \multirow[t]{2}{*}{$67-63-0$} \\
\hline Isopropyl alcohol (dimer) & & 1,000 & 1.64 & \\
\hline Methyl salicylate & Positive & 40 & 1.69 & \multirow{2}{*}{$119-36-8$} \\
\hline Methyl salicylate & Negative & 260 & 1.53 & \\
\hline Sevoflurane (monomer) & \multirow{2}{*}{ Negative } & 50 & 1.56 & \multirow{2}{*}{$28523-86-6$} \\
\hline Sevoflurane (dimer) & & 1,500 & 1.22 & \\
\hline
\end{tabular}




\section{Conclusion}

In this work, we investigated the analytical performance of an IMS with orthogonally mounted X-ray source. Therefore, our existing IMS design was modified to couple an X-ray source both axially and orthogonally for direct comparison. As shown in our previous work, an axially mounted X-ray source leads to an ionization in the drift region and thus to an increasing offset current and Fano noise. The presented orthogonal mounting of the X-ray source now focuses the ionization mainly to the ionization region. As a result, the offset current and noise significantly decrease. The considerably improved SNR and the associated reduced detection limits now enable a highly sensitive IMS with a non-radioactive ionization source. This not only reduces legal restrictions affecting sales, transport and disposal, but also gives high sensitivity and control of the ionization source. The LoDs in the low ppt $\mathrm{v}_{v}$ range in one second for hazardous substances. Furthermore, pulsed ionization is possible by controlling the $\mathrm{X}$-ray source through additional electronics to be developed. This will allow ion generation and recombination measurements.

\section{ACKNOWLEDGMENT}

We thank Arben Pulaj and Alexander Labohm for their help during this work.

\section{REFERENCES}

[1] G.A. Eiceman, Z. Karpas, H.H. Hill, Ion mobility spectrometry, 3rd ed., CRC Press, Boca Raton, 2013.

[2] G.A. Eiceman, A.P. Snyder, D.A. Blyth, Monitoring of Airborne Organic Vapors Using Ion Mobility Spectrometry, International Journal of Environmental Analytical Chemistry 38 (1990) 415-425.

[3] A.B. Kanu, H.H. Hill, Identity confirmation of drugs and explosives in ion mobility spectrometry using a secondary drift gas, Talanta 73 (2007) 692-699.

[4] R.G. Ewing, D.A. Atkinson, G.A. Eiceman, G.J. Ewing, A critical review of ion mobility spectrometry for the detection of explosives and explosive related compounds, Talanta 54 (2001) 515-529.

[5] C. Wu, W.E. Steiner, P.S. Tornatore, L.M. Matz, W.F. Siems, D.A. Atkinson, H.H. Hill, Construction and characterization of a high-flow, high-resolution ion mobility spectrometer for detection of explosives after personnel portal sampling, Talanta 57 (2002) 123-134.

[6] J.I. Baumbach, S. Sielemann, Z. Xie, H. Schmidt, Detection of the Gasoline Components Methyl tert Butyl Ether, Benzene, Toluene, and $\mathrm{m}$-Xylene Using Ion Mobility Spectrometers with a Radioactive and UV Ionization Source, Anal. Chem. 75 (2003) 1483-1490.

[7] St. Sielemann, J.I. Baumbach, H. Schmidt, P. Pilzecker, Detection of alcohols using UV-ion mobility spetrometers, Analytica chimica acta 431 (2001) 293-301.

[8] H. Borsdorf, Determination of n-alkanes and branched chain alkanes by Corona discharge ion mobility spectrometry, Int. J. Ion Mobil. Spec. 1999 9-14.

[9] C.L. Crawford, H.H. Hill, Comparison of reactant and analyte ions for ${ }^{63} \mathrm{Nickel}$, corona discharge, and secondary electrospray ionization sources with ion mobility-mass spectrometry, Talanta 107 (2013) 225-232.

[10] G.A. Eiceman, J.H. Kremer, A.P. Snyder, J.K. Tofferi, Quantitative Assessment of a Corona Discharge lon Source in Atmospheric Pressure Ionization-Mass Spectrometry for Ambient Air Monitoring, International Journal of Environmental Analytical Chemistry 33 (1988) 161-183.

[11] P. Cochems, A.T. Kirk, E. Bunert, M. Runge, P. Goncalves, S. Zimmermann, Fast pulsed operation of a small non-radioactive electron source with continuous emission current control, Rev. Sci. Instrum. 86 (2015) 65102. 
[12] P. Cochems, M. Runge, S. Zimmermann, A current controlled miniaturized non-radioactive electron emitter for atmospheric pressure chemical ionization based on thermionic emission, Sensors and Actuators A: Physical 206 (2014) 165-170.

[13] T. Reinecke, A.T. Kirk, A. Heptner, D. Niebuhr, S. Bottger, S. Zimmermann, A compact high-resolution Xray ion mobility spectrometer, Rev. Sci. Instrum. 87 (2016) 53120.

[14] A.T. Kirk, M. Allers, P. Cochems, J. Langejuergen, S. Zimmermann, A compact high resolution ion mobility spectrometer for fast trace gas analysis, Analyst 138 (2013) 5200-5207.

[15] A. Kuklya, T. Reinecke, F. Uteschil, K. Kerpen, S. Zimmermann, U. Telgheder, X-ray ionization differential ion mobility spectrometry, Talanta 162 (2017) 159-166.

[16] U. Fano, Ionization Yield of Radiations. II. The Fluctuations of the Number of lons, Phys. Rev. 72 (1947) 26-29.

[17] A.T. Kirk, S. Zimmermann, Pushing a compact $15 \mathrm{~cm}$ long ultra-high resolution drift tube ion mobility spectrometer with $R=250$ to $R=425$ using peak deconvolution, Int. J. Ion Mobil. Spec. 18 (2015) 1722.

[18] A.T. Kirk, S. Zimmermann, Bradbury-Nielsen vs. Field switching shutters for high resolution drift tube ion mobility spectrometers, Int. J. Ion Mobil. Spec. 17 (2014) 131-137.

[19] E.A. Mason, E.W. McDaniel, Transport Properties of lons in Gases, Wiley-VCH Verlag GmbH \& Co. KGaA, Weinheim, FRG, 1988.

[20] A.B. Kanu, M.M. Gribb, H.H. Hill, Predicting optimal resolving power for ambient pressure ion mobility spectrometry, Anal. Chem. 80 (2008) 6610-6619. 